\title{
Public Title
}

National Cancer Institute

\section{Source}

National Cancer Institute. Public Title. NCI Thesaurus. Code C93637.

The title of the document intended for the general population. 